\title{
An overview of turbulence compensation
}

\author{
Klamer Schutte ${ }^{1 *}$, Adam W.M. van Eekeren ${ }^{1}$, Judith Dijk ${ }^{1}$, \\ Piet B.W. Schwering ${ }^{1}$, Miranda van Iersel ${ }^{1}$, Niek J. Doelman ${ }^{2}$ \\ ${ }^{1}$ TNO, PO Box 96864, 2509 JG The Hague, The Netherlands \\ ${ }^{2}$ TNO, PO Box 155, 2600 AD Delft, The Netherlands
}

\begin{abstract}
In general, long range visual detection, recognition and identification are hampered by turbulence caused by atmospheric conditions. Much research has been devoted to the field of turbulence compensation. One of the main advantages of turbulence compensation is that it enables visual identification over larger distances. In many (military) scenarios this is of crucial importance. In this paper we give an overview of several software and hardware approaches to compensate for the visual artifacts caused by turbulence. These approaches are very diverse and range from the use of dedicated hardware, such as adaptive optics, to the use of software methods, such as deconvolution and lucky imaging. For each approach the pros and cons are given and it is indicated for which type of scenario this approach is useful. In more detail we describe the turbulence compensation methods TNO has developed in the last years and place them in the context of the different turbulence compensation approaches and TNO's turbulence compensation roadmap. Furthermore we look forward and indicate the upcoming challenges in the field of turbulence compensation.
\end{abstract}

Keywords: Turbulence compensation, image restoration, deconvolution, adaptive optics, phase diversity, lucky imaging

\section{INTRODUCTION}

In long range recognition atmospheric conditions play an important role. Beside transmission effects hampering the detection of a target signal over a long range, turbulence influences the image of a target over long ranges. In most long range conditions image resolution is limited by atmospheric conditions. The nature of the atmospheric wavefront perturbations is described by the Kolmogorov model, developed further by Tatarski [1, 2]. The model assumes that the wavefront perturbations are caused by variations in the refractive index of the atmosphere, resulting in phase fluctuations. In this model, the refractive-index structure parameter $C_{n}{ }^{2}\left[\mathrm{~m}^{-2 / 3}\right]$, and its variation, determine the turbulence effects over the path. In particular near the surface large values of $C_{n}{ }^{2}$ and large fluctuations occur, dominating the image resolution.

Another important parameter used in the field of atmospheric turbulence is the so-called Fried's seeing diameter (the Fried parameter, $\left.r_{0}[\mathrm{~m}]\right)$. This value describes the dimension of a hypothetical telescope with which the atmospheric turbulence is measuring the scene. When $r_{0}$ is less than the system aperture $D$, the image resolution is limited by the atmospheric turbulence (i.e. $D / r_{0}>1$ ). For a point source the spherical wave approximation yields:

$$
r_{0}=\left[0.423 k^{2} \int_{0}^{L} C_{N}^{2}(z)\left(\frac{z}{L}\right)^{5 / 3} d z\right]^{-3 / 5},
$$

with $L$ the optical path length over which $z$ integrates and $k=2 \pi / \lambda$ the wavenumber. The isoplanatic angle is the angle within which the phase shift is limited to about 1 radian. Within this angle the optical path length does not differ significantly from the path length in the optical centre. This angle is given by:

$$
\theta_{0}=\left[2.91 k^{2} L^{5 / 3} \int_{0}^{L} C_{N}^{2}(z)\left(1-\frac{z}{L}\right)^{5 / 3} d z\right]^{-5 / 3} .
$$

\footnotetext{
*klamer.schutte@tno.nl; phone +31 8886 64109; www.tno.nl
} 
Over long horizontal paths the values of $\theta_{0}$ are typically very small. In the case of small isoplanatic angles, turbulence corrections are required in each image patch of size of the isoplanatic angle. An advanced study in characterizing the turbulence over a long period of time is presented in [3]. The Fried parameter was used in describing the average Modulation Transfer Function (MTF) of images taken through the atmosphere. The imagery can be distinguished in three regimes: long-exposure imagery, short-exposure imagery without scintillation, and short-exposure imagery with scintillation. The main distinction between short and long exposure lies in the correction of atmospheric tilt. In long exposures the image centre wanders randomly and fast over the image plane.

To improve the detection, recognition and identification range, it is very important to compensate for the visual degradation in images caused by atmospheric turbulence. Turbulence compensation is a challenging problem because 1) the amount of turbulence is spatially varying due to anisoplanatic conditions while the isoplanatic angle, i.e. the angle over which the turbulence can be assumed constant, varies with atmospheric conditions and 2) the amount of turbulence varies significantly in time. According to Roggeman and Welsh [4] there are basically three different approaches to compensate for turbulence effects in images: 1) adaptive optics techniques (hardware), 2) post-processing techniques (software) and 3) a mixture of both techniques. In the following subsections each approach is briefly described.

\subsection{Hardware approach}

A hardware approach is often used within astronomy applications. Except for space telescopes, all high-end telescopes are hindered by the Earth's atmosphere. Modern telescopes typically include an adaptive optics part, which corrects the atmospheric distortion [5]. A typical assumption in such a system is that the turbulence effect is constant over the field of view of the telescope. For astronomy applications the imaging path is an almost vertical path through the atmosphere and effectively resulting in one or two phase screens. However, for ground-to-ground applications the full imaging path is a near horizontal path through the atmosphere, which has likely many variations of the refractive index. This will result in a more complex turbulence setup with multiple phase screens. In effect, the isoplanatic angle will often be much smaller than the field of view of the camera system, up to the level where it is even smaller than the IFOV (Instanteneous Field Of View, i.e. opening angle per pixel). This means that a closed loop adaptive optics system, such as used in astronomy applications, is not suitable for ground-to-ground imaging.

\subsection{Software approach}

The second approach is a software-based approach where the images are processed (preferably in real-time) after recording. There exist many different algorithms which can be divided into the following basic categories: global and local processing. Local processing can be done on a spatial and/or temporal level. A global processing approach is described in [6], where a global registration and deconvolution is done and all frames are processed. Such an approach is useful for images captured under weak turbulence conditions. When the turbulence conditions are stronger (and the isoplanatic angle is smaller) the variations within and between the frames are larger. This requires a local processing approach, such as described in [7, 8, 9]. Promising is the combination with Super Resolution processing where turbulence induced blur can be handled similar to optical blur [10]. A well-known local temporal approach is luckyimaging $[11,12,13]$. Here, the 'lucky' (best quality) frames are automatically selected for processing. While lucky imaging approaches can obtain very good results, its downside is the amount of time needed before a sufficient good image patch is seen for every image position. Promising here is the use of phase diversity imaging [14, 15] (a mixture of hardware and software approach as will be explained in the next section) which significantly improves the time to observe good image patches.

\subsection{Mixture of hardware and software approach}

Phase diversity is a typical mixture approach in which dedicated hardware is used in combination with post-processing (software). Phase diversity does not record a single image, but several (often two) images simultaneously. These different images differ by a known phase aberration, such as a defocus. Having multiple images allows recovering its phase components. In turn, knowing its phase, allows to truly correct any distortion, where the earlier discussed approaches typically resort to a phase-free blind deconvolution deblur approach. Similar to such deblurring approaches the phase diversity approach can be applied to non-isoplanatic imaging conditions [16, 17]. An extension to the above 
described phase diversity approach is described in [18]. This approach aims to use, instead of 'basic' phase diversity, only one optical path containing a wavefront modulator which sweeps rapidly through the aberration-configuration space. A larger sweep through the aberration configuration space translates to higher turbulence which can be corrected. When its scan rate is higher than the rate of the atmospheric turbulence, this guarantees that 'lucky' images are seen. A disadvantage of this approach is that the modulations must be generated and recorded really fast and therefore expensive hardware (wavefront modulator) is needed.

\subsection{Contents of this paper}

In this paper we give an overview of several software and hardware approaches to compensate for the visual artifacts caused by turbulence. Section 2 describes the TNO roadmap for turbulence compensation in relation with the different turbulence compensation approaches. In Section 3 different turbulence compensation methods developed by TNO are described. Finally, conclusions and future challenges will be discussed in Section 4.

\section{TNO TURBULENCE COMPENSATION ROADMAP}

Within TNO there is much knowledge [19] about atmospheric turbulence and turbulence compensation methods. In recent years several methods have been developed to compensate for the effects of turbulence [18, 7, 6, 20]. In this section these methods will be placed in the context of the different approaches (hardware-, software- and mixture approach) as discussed in the introduction. To give more insight into how turbulence compensation methods interact with the observed imagery, we ascribe the following 'properties' to turbulence compensation methods: 1) spatial approach, 2) processing approach and 3) optical setup. These 'properties' are depicted in Figure 1. The property spatial approach indicates if each frame is processed spatially at a global or local scale. With processing approach the mode of processing is indicated, which can be 'using all images' or 'selecting a lucky image' or 'estimating the correct wavefront'. The last property indicates the optical setup, which can be 'normal' or 'using a wavefront modulator'.

In Figure 1 the connection between the properties for an adaptive optics (hardware) approach is indicated with a solid line. Such an approach uses a wavefront modulator with feedback (adaptive optics) and it tries to correct all frames at a global scale (full field-of-view).

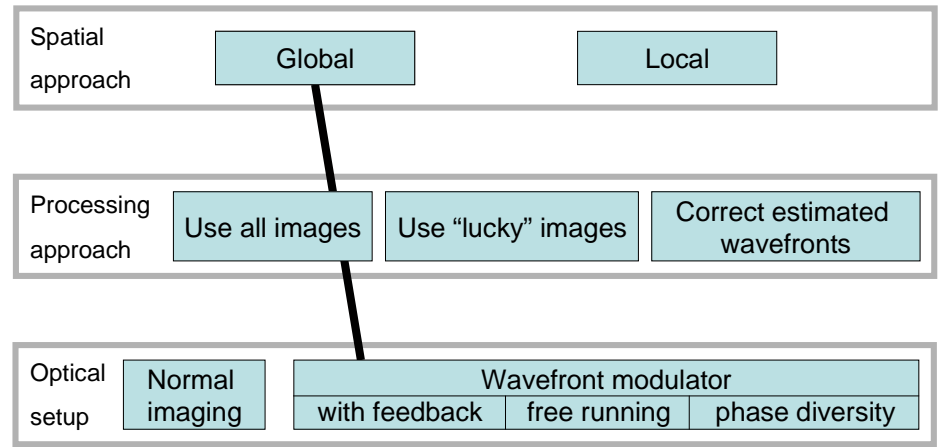

Figure 1. Turbulence compensation properties of a typical hardware approach (adaptive optics). The connection is indicated with a solid line and will be explained in more detail in Section 3.1.

In Figure 2 a few software approaches that are developed within TNO are mapped on the turbulence compensation properties. The connections are indicated with a solid line. The left line represents a deconvolution method at a global scale (see for more details Section 3.2) and the right line represents a deconvolution method that processes at a local scale and uses lucky images (see for more details Section 3.3). Of course other approaches are possible as well, e.g. a method that uses a local spatial approach, uses all images and has a normal imaging optical setup. The drawback of this approach (in comparison with the right thick line) is that low quality frames will have a negative effect on the result after processing. 


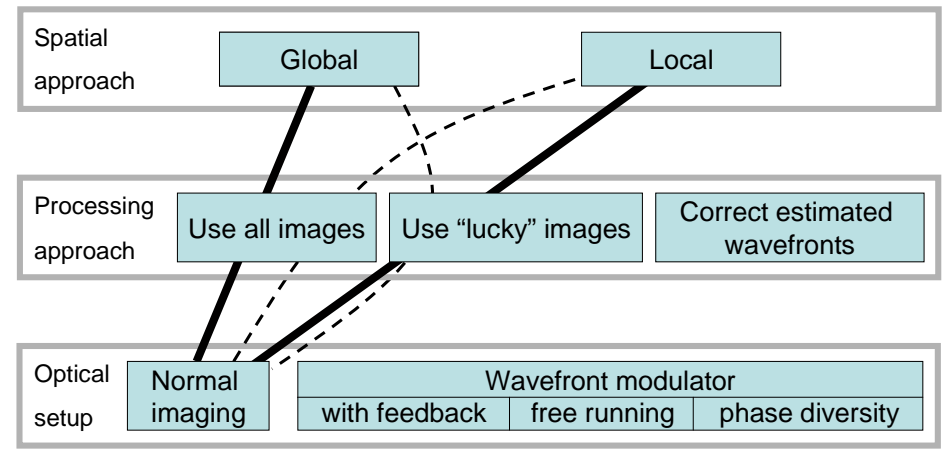

Figure 2. Turbulence compensation properties of a few software approaches. The connections are indicated with a solid line. The left line represents a deconvolution method at a global scale and the right line represents a deconvolution method that processes at a local scale and uses lucky images.

In Figure 3 a few mixtures of hardware and software approaches are depicted. The solid line depicts the method developed by TNO and it uses a free-running wavefront modulator to estimate the correct wavefront by post processing (see for more details Section 3.4). The dashed line on the left is a variation on this method using lucky images and the dashed line on the right indicates the well-known phase diversity approach.

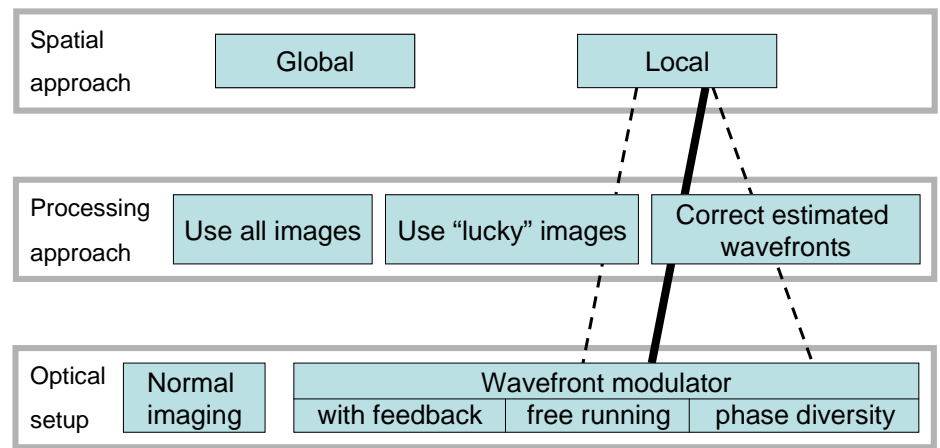

Figure 3. Turbulence compensation properties of a few mixture approaches. The connection indicated with a solid line represents a method developed by TNO. The dashed lines represent a variation on this method (left line) and the wellknown phase diversity approach (right line).

The focus of the TNO turbulence compensation roadmap is to develop software- and hardware-based methods that can cope with imagery captured under weak to medium turbulence conditions. A local approach (spatial and temporal) is necessary to compensate for these conditions. The software-based methods must be low-cost, easy to implement and preferably working in real-time. The hardware-based methods will be further developed to meet the challenging astronomy requirements of wide-field imaging with laser guide stars and high contrast extrasolar planet detection. On the longer term an integration of the hardware- and software-based methods is foreseen, in which the actions of optical wavefield manipulation and image restoration are matched such that the overall, resultant image quality is optimized. 


\section{TNO TURBULENCE COMPENSATION METHODS}

In the last decennium TNO has developed several methods for turbulence compensation. The following subsections describe the different methods as mentioned in the previous section.

\subsection{Hardware approach: Adaptive optics}

The technique of Adaptive Optics aims at the real-time and optical compensation of the turbulence effects by using a deformable mirror, a wavefront sensor and a real-time feedback controller. For fields of view larger than the isoplanatic angle a system with multiple deformable mirrors and wavefront sensors can be employed. In the astronomy application natural guide stars or laser guide stars are often used as a bright reference source to obtain wavefront aberration correction for a nearby faint science object. In recent years TNO has been focusing on several components of an Adaptive Optics system, i.e. novel deformable mirror technology, improvement of the real-time control performance and launch telescope systems for laser guide stars.

In co-operation with the Eindhoven University of Technology a modular deformable mirror technology has been developed based on electro-magnetic actuators (see left part of Figure 4). Prototypes of the mirror consist of a $0.1 \mathrm{~mm}$ thick, continuous facesheet on which low voltage, variable reluctance type, push-pull actuators impose out-of-plane displacements. The thin facesheet is selected for its low moving mass and low out-of-plane stiffness, thereby preventing large power dissipation and the need for active cooling. The variable reluctance actuators are located in an actuator module. The module holds 61 actuators; each actuator has $\pm 10 \mu \mathrm{m}$ stroke and nanometer resolution. Each actuator module is driven by a printed circuit board (PCB) with dedicated driver electronics. The PCB's are connected by a full duplex multi-drop low voltage differential signaling (LVDS) cable. The Ethernet-LVDS bridge enables any commercial PC to control the deformable mirror using standard protocols. With the actuator module and accompanying electronics the mirror system is modular both in mechanics and electronics.

The real-time control performance of Adaptive Optics systems can be improved significantly by the application of optimality-based control approaches. TNO has studied and developed an H2-optimal control approach which accounts for the specific temporal and spatial correlation properties of turbulence-induced wavefront aberrations. The main philosophy behind this approach is that the inherent, Kalman-type wavefront prediction is fully data driven. This means that no initial assumptions are made on properties such as the spatial correlation, modal decoupling, possibly frozen turbulence flow or the (low) order of disturbance models. The design of the optimal controller is completely determined by the statistics of the measured wavefront sensor signals. This control approach has been validated in the laboratory on an experimental AO set-up and also on a solar telescope with true on-sky turbulence. The experiment on the McMathPierce telescope demonstrates the significant performance improvement of the optimal controller over the commonly used integrator controller for the low order modes [21].
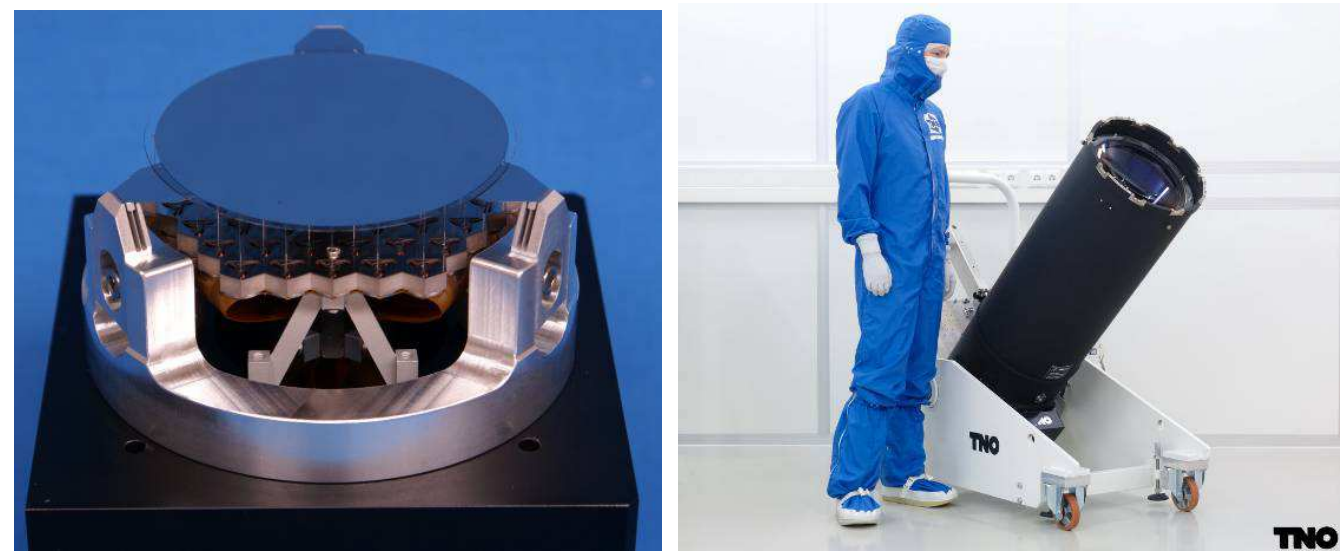

Figure 4. (a) Prototype of a 61 actuators deformable mirror. (b) Laser launch telescope system as developed for the ESO's Adaptive Optics facility at the VLT. 
To increase the sky coverage of ESO's new Adaptive Optics facility on the Unit Telescope 4 of the Very Large Telescope (VLT), four laser guide star facilities will be installed. TNO has developed the Launch Telescope System for the laser guide star facility. These launchers are Galilean beam expanders, expanding a $\varnothing 15 \mathrm{~mm}$ input beam to a steerable $\varnothing 300 \mathrm{~mm}$ output beam with a wavefront quality requirement of $<50 \mathrm{~nm} \mathrm{rms}$. This wavefront quality needs to be maintained in the presence of environmental air temperature fluctuations. The allowed defocus for instance is only 0.2 waves under a $-0.7^{\circ} \mathrm{C} / \mathrm{hr}$ temperature gradient in an operational range of $0-15^{\circ} \mathrm{C}$. The thermal behaviour of the system has been analyzed by combining optical, lumped mass and FE analyses. The design is passively a-thermalized over a large temperature range as well as under the influence of thermal gradients. Extensive thermal and high power laser testing has shown that the system performs as required [22].

\subsection{Global-based software approach}

This software approach is based on earlier developed image enhancement tools as described in [23, 20]. First, the images in the original sequence are registered to a reference frame. In the next step a deconvolution is performed on this stabilized sequence. For this step several deconvolution algorithms were tested. After this deblurring step the image enhancement tools were used to visualize more details. A result of this method is shown in Figure 5, where an image sequence of a ship at a distance of approximately $12.5 \mathrm{~km}$ is used. In the steps mentioned above, all frames in the sequence were taken into account.

As can be seen in Figure 5, an advantage of this method is a significant sharpening of details lost in the original by the atmospheric turbulence. Another advantage is that the processing can be done in real-time. A disadvantage of this method is that the deconvolution is performed on a global scale, i.e., on the whole image. Images captured under stronger turbulence conditions can show variations within an image and between images that are too large to process correctly using a global based approach.

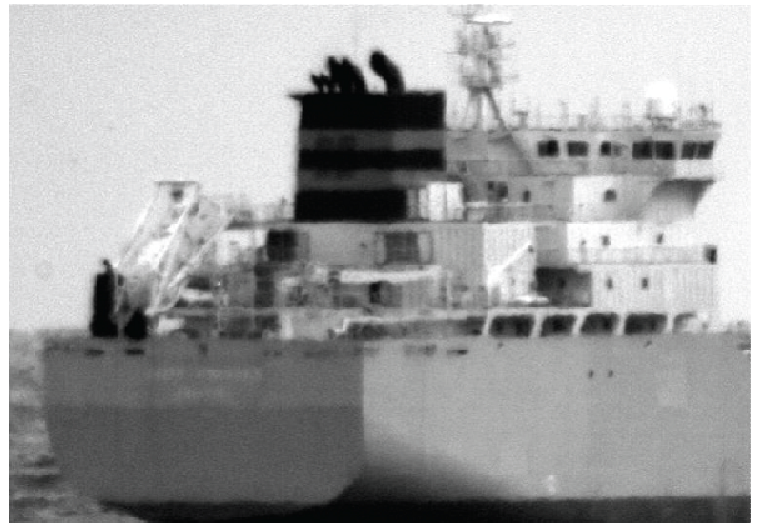

(a)

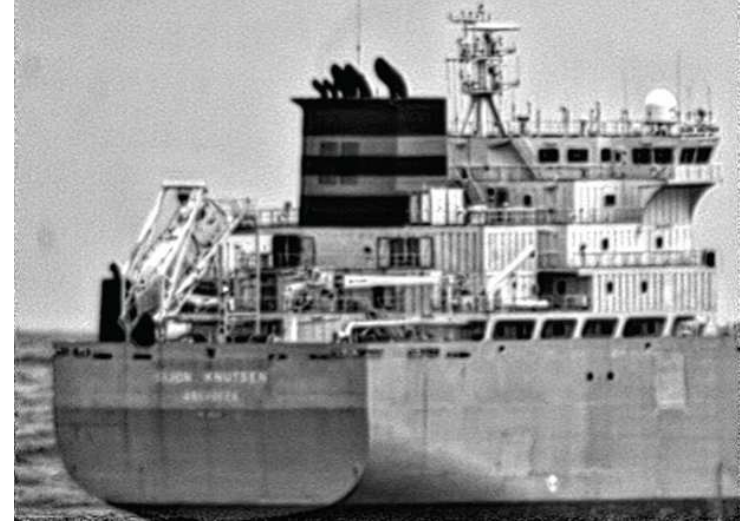

(b)

Figure 5. (a) One frame of a turbulence deteriorated image sequence. (b) One frame of the processed sequence using TNO's global-based turbulence compensation method [6].

\subsection{Local-based software approach}

Because a phase diversity approach requires complex and expensive hardware, recently a software based method is developed which performs local-based turbulence compensation on raw input data. The local-based aspect of this method is that the motion compensation is done on a local scale. Basically the method consists of the following parts: 1) calculation of a reference frame, 2) frame selection based on maximum gradient magnitude, 3) global and local motion compensation and 4) multi-frame deblurring. A nice feature of the latter part is that also resolution enhancement is possible. The method described above is also able to perform well on data which contains moving objects. A more detailed description can be found in [7]. An example of the capability of this method using resolution enhancement is depicted in Figure 6. It is clear that in the processed frame the words "Sea Rescue" are much better visible. The raw 
imagery was captured during the FATMOSE trial in False Bay, South Africa in 2009 - 2010. The frame shown in Figure 6 (a) was captured at 20 April 2010 at $16.35 \mathrm{~h}$ under weak turbulence conditions $\left(\mathrm{C}_{\mathrm{n}}^{2}=2.9 \times 10^{-16} \mathrm{~m}^{-2 / 3}\right)$ at a range of 15.7 $\mathrm{km}$. More details about the FATMOSE trial can be found in [24].

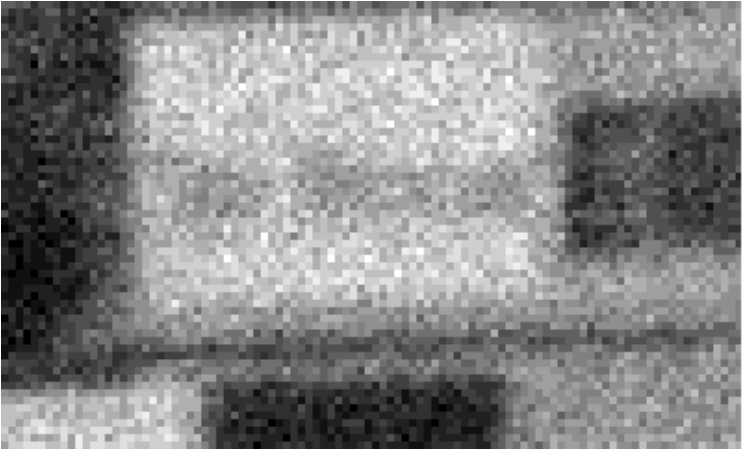

(a)

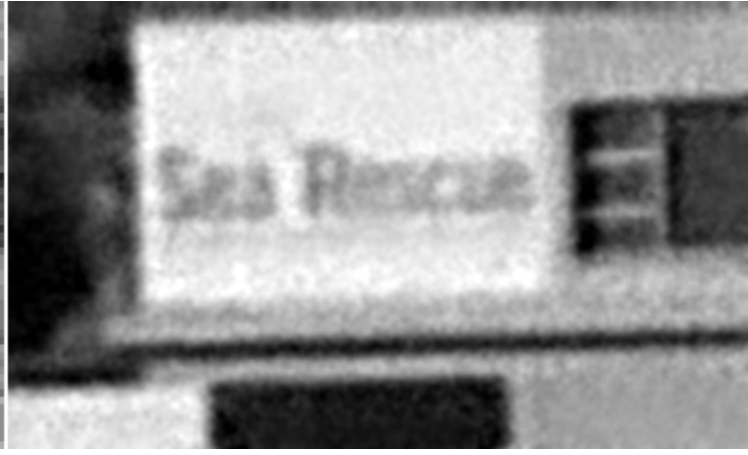

(b)

Figure 6. (a) Region-of-interest of deteriorated input frame 100. (b) Resulting frame after processing with resampling factor 4 and local contrast enhancement applied [25] for better visualisation.

Another result is shown in Figure 7 after processing a sequence containing more local turbulence artifacts. This sequence was captured during a NATO SET-165 trial in Dayton, USA in October 2011 [26]. The frame shown in Figure 7 (a) was captured at 12 October 2011 at $15.35 \mathrm{~h}$ under weak turbulence conditions $\left(\mathrm{C}_{\mathrm{n}}{ }^{2}=6.9 \times 10^{-16} \mathrm{~m}^{-2 / 3}\right)$ at a range of approximately $7 \mathrm{~km}$. It is clear that more detail is visible in the processed frame, such as on the checkerboard and the NATO sign on the building. The stabilizing effect of this method can be appreciated best by watching a video.

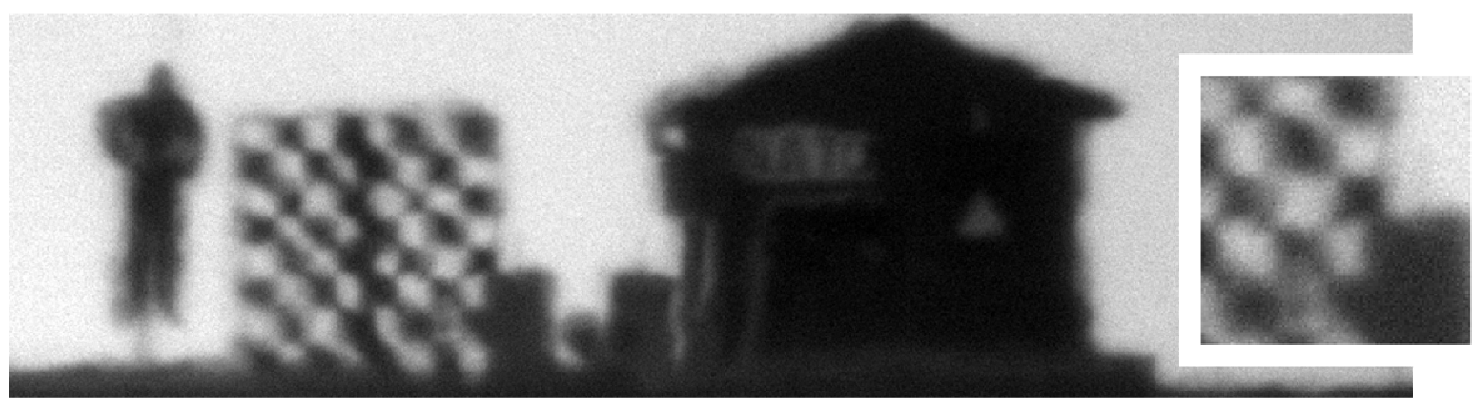

(a)

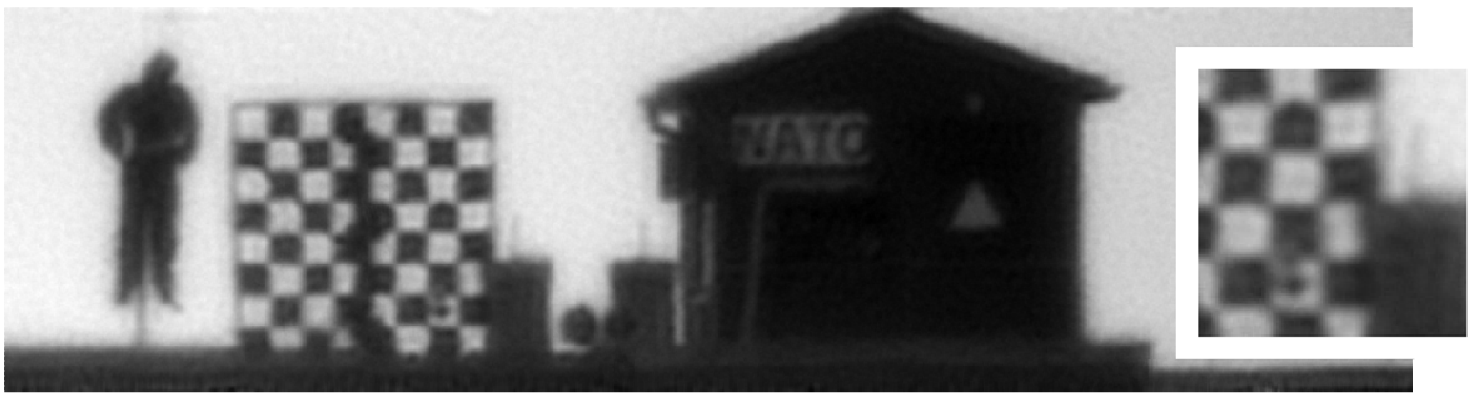

(b)

Figure 7. (a) One of the deteriorated frames of the "NATO" sequence taken on 12 October 2011 at $15.35 \mathrm{~h}$ with on the right side a detail of the checkerboard. (b) Corresponding processed frame by the local-based method. 


\subsection{Mixture approach: Free running wavefront modulator}

The principle of a this approach is that by means of a free-running wavefront modulator a set of known phase front aberrations is made to an incoming wavefront. Because the phase front aberrations are known, it is possible to estimate the phase of the incoming wavefront and thereby calculate the corresponding turbulence free image. A more detailed description of this method can be found in [18]. In Figure 8 an example is depicted of a turbulence deteriorated image (a) which is deblurred using the additional phase diversity images (b)-(d). The resulting enhanced image is depicted in (f).

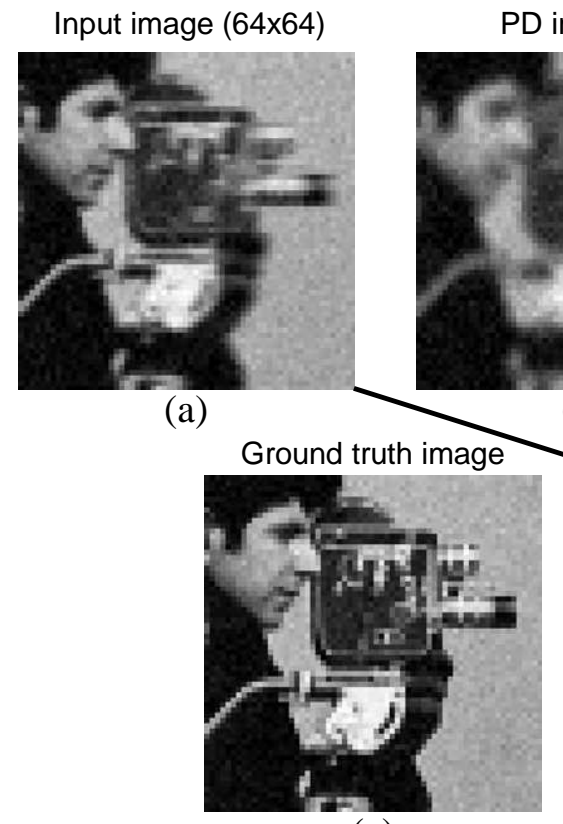

(e)
PD image 1

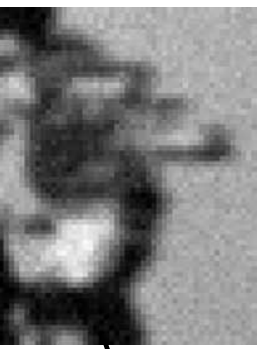

(b)

Estimated image

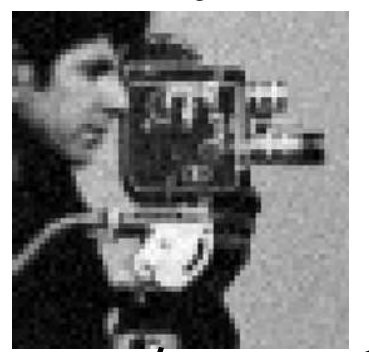

(c)

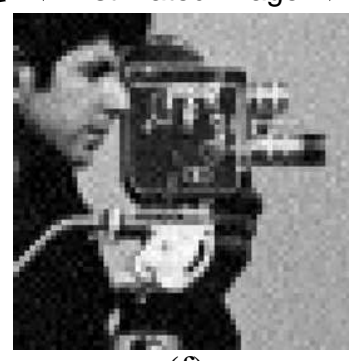

(f)

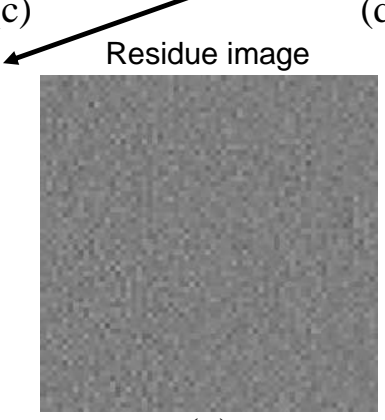

(g)
PD image 3

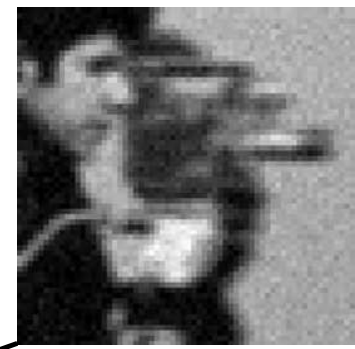

(d)

Figure 8. Example of turbulence compensation using TNO's phase diversity approach. (a) Input image $[0,1]$ with spatially varying defocus (increasing from left to right), (b)-(d) Three different phase-diversity images, (e) Ground truth image, (f) Estimated image after turbulence compensation using phase diversity, (g) Residue [-0.5, 0.5] between (e) and (f).

When a wavefront modulator is able to sweep very fast through many phase modulations it is possible to process the imagery on a local scale, i.e. the chance that a lucky sample is observed increases. This property makes this approach also suitable when real-time turbulence compensation is required, such as when moving targets are observed.

\section{CONCLUSIONS AND FUTURE CHALLENGES}

In the last decennium TNO has developed hardware and software for turbulence compensation, which can be used for different scenarios ranging from astronomy to (military) ground-to-ground scenarios. From our experience the following conclusions can be drawn:

- The effects of weak turbulence conditions can be compensated by processing on a global scale. Such processing can be done in real-time by hardware as well as software.

- The effects of weak to medium turbulence conditions can be compensated by processing on a local scale. This can be done by using additional hardware (phase diversity) or by using a local-based software approach. 
- Phase diversity has the advantage that the turbulence effects can be compensated in each frame without losing temporal resolution. The drawback is that it needs more complex optical hardware.

- A local-based software approach is a more affordable alternative for phase diversity. Drawbacks are the demanding processing and the decrease of temporal resolution.

Although a lot of advances are made in the field of turbulence compensation in recent years, there remain a lot of challenges as well. A major challenge is to develop more advanced local-based methods that are capable of 1) estimating and compensating blur on a local level [27], 2) estimating and compensating motion on a local level, 3) selecting the best temporal information on a local level and 4) performing previous capabilities on static and dynamic objects. Future work is needed to handle medium to high turbulence conditions. Where in astronomy the state of the art applies adaptive optics, we need to look into multiple adaptive optics solutions for ground to ground scenarios such as the time-varying phase diversity setup.

\section{REFERENCES}

[1] Roddier, F., [Diffraction Limited Imaging with Very Large Telescopes], ch. Propagation and Image Formation through the Turbulent Atmosphere, 33 - 52, Kluwer (1989).

[2] Schmidt, J., [Numerical Simulation of Optical Wave Propagation], ch. 9, SPIE, Bellingham (2010).

[3] de Jong, A., Schwering, P., Benoist, K., Gunter, W., Vrahimis, G., and October, F., "Long-term measurements of atmospheric point-spread functions over littoral waters as determined by atmospheric turbulence," Proc. SPIE Infrared Imaging Systems: Design, Analysis, Modeling, and Testing XXIII 8355 (2012).

[4] Roggeman, M. and Welsh, B., "Imaging through turbulence," CRC Press, Boca Raton, USA (1996).

[5] Roddier, F., ed., [Adaptive Optics in Astronomy], Cambridge University Press (1999).

[6] van Iersel, M. and van Eijk, A. M. J., "Estimating turbulence in images," Proc. SPIE Free-Space Laser Communications X 7814 (2010).

[7] van Eekeren, A. W. M., Kruithof, M. C., Schutte, K., Dijk, J., van Iersel, M., and Schwering, P. B. W., "Patch-based local turbulence compensation in anisoplanatic conditions," Proc. SPIE Infrared Imaging Systems: design, analysis, modeling and testing XXIII 8355 (2012).

[8] Huebner, C. S., "Compensating image degradation due to atmospheric turbulence in anisoplanatic conditions," Proc. SPIE Mobile Multimedia/Image Processing, Security, and Applications 7351 (2009).

[9] Zhu, X. and Milanfar, P., "Image reconstruction from videos distorted by atmospheric turbulence," Proc. SPIE Visual Information Processing and Communication 7543 (2010).

[10] Pham, T., van Vliet, L., and Schutte, K., "Influence of signal-to-noise ratio and point spread function on limits of super-resolution," Proc. SPIE Image Processing: Algorithms and Systems IV 5672 (2005).

[11] Aubailly, M., Vorontsov, M. A., Carhart, G. W., and Valley, M. T., "Automated video enhancement from a stream of atmospherically-distorted images: the lucky-region fusion approach," Proc. SPIE Atmospheric Optics 7463 (2009).

[12] Fried, D., "Probability of getting a lucky short-exposure image through turbulence," Optical Society of America Journal 68, 1651-1658 (1978).

[13] Woods, S. C., Burnett, J. G., Kent, P. J., and Turner, A. J., "High-resolution imaging using lucky frame selection," Defence Codex, The magazine for Defence Engineering and Science 2, 1-12 (2008).

[14] Gonsalves, R. A., "Phase retrieval and diversity in adaptive optics," Optical Engineering 21(5), 829-832 (1982).

[15] Korkiakoski, V., Keller, C., Doelman, N., Fraanje, R., and Verhaegen, M., "Joint-optimization of phasediversity and adaptive optics: demonstration of potential," Applied Optics 51, 102 - 113 (2012).

[16] Gonsalves, R. A., "Nonisoplanatic imaging by phase diversity," Optics Letters 19(7), 493-495 (1994).

[17] Paxman, R. G., Thelen, B. J., and Seldin, J. H., "Phase-diversity correction of turbulence-induced spacevariant blur," Optics Letters 19(16), 1231-1233 (1994).

[18] van Eekeren, A. W. M., Schutte, K., Dijk, J., and Schwering, P. B. W., "Time-varying phase diversity turbulence compensation," Proc. SPIE Infrared Technology and Applications XXXVII 8012 (2011). 
[19] van Eekeren, A., Schutte, K., Dijk, J., Schwering, P., van Iersel, M., and Doelman, N., "Turbulence compensation: an overview," Proc. SPIE Infrared Imaging Systems: Design, Analysis, Modeling, and Testing XXIII 8355 (2012).

[20] Schwering, P. B. W., van den Broek, S. P., and van Iersel, M., "EO system concepts in the littoral," Proc. SPIE Infrared Technology and Applications XXXIII 6542 (2007).

[21] Doelman, N. and et al., "Optimal control of tip-tilt modes - on sky adaptive optics demonstration," Proc. of the AO4ELT 2 Conference, Victoria (2011).

[22] Kamphues, F. and et al., "Athermal laser launch telescopes," Proc. of the AO4ELT 2 Conference, Victoria (2011).

[23] Schutte, K., de Lange, D., and van den Broek, S., "Signal conditioning algorithms for enhanced tactical sensor imagery," Proc. SPIE Infrared Imaging Systems: Design, Analysis, Modeling, and Testing XIV 5076 (2003).

[24] de Jong, A., Fritz, P., Benoist, K., van Eijk, A., and Schwering, P., "Preliminary results of the FATMOSE atmospheric propagation trials in the False Bay, South Africa, November 2009 - July 2010," Proc. SPIE Optics in Atmospheric Propagation and Adaptive Systems XIII 7828 (2010).

[25] Schutte, K., "Multiscale adaptive gain control of IR images," Proc. SPIE Infrared Technology and Applications XXIII 3061 (1997).

[26] Velluet, M., Vorontsov, M., Espinola, R., Marchi, G., Nicolas, S., Schwering, P., and Riker, J., "Turbulence characterization and image processing data sets from a NATO RTO SET 165 trial in Dayton, OH, USA," Proc. SPIE Atmospheric Propagation IX 8380 (2012).

[27] Bouma, H., Dijk, J., and van Eekeren, A., "Precise local blur estimation based on the first-order derivative," Proc. SPIE Visual Information Processing XXI 8399 (2012). 